Vulcan Petawatt—an ultra-high-intensity interaction facility

This article has been downloaded from IOPscience. Please scroll down to see the full text article. 2004 Nucl. Fusion 44 S239

(http://iopscience.iop.org/0029-5515/44/12/S15)

View the table of contents for this issue, or go to the journal homepage for more

Download details:

IP Address: 130.246.132.178

The article was downloaded on 13/01/2012 at 09:44

Please note that terms and conditions apply. 


\title{
Vulcan Petawatt—an ultra-high-intensity interaction facility
}

\author{
C.N. Danson, P.A. Brummitt, R.J. Clarke, J.L. Collier, B. Fell, \\ A.J. Frackiewicz, S. Hancock, S. Hawkes, C. Hernandez-Gomez, \\ P. Holligan, M.H.R. Hutchinson, A. Kidd, W.J. Lester, \\ I.O. Musgrave, D. Neely, D.R. Neville, P.A. Norreys, D.A. Pepler, \\ C.J. Reason, W. Shaikh, T.B. Winstone, R.W.W. Wyatt and \\ B.E. Wyborn \\ CCLRC Rutherford Appleton Laboratory, Chilton, Didcot, Oxfordshire, OX11 0QX, UK \\ E-mail: c.danson@rl.ac.uk
}

Received 31 March 2004, accepted for publication 17 August 2004

Published 26 November 2004

Online at stacks.iop.org/NF/44/S239

doi:10.1088/0029-5515/44/12/S15

\begin{abstract}
The Vulcan Nd: glass laser at the Central Laser Facility is a Petawatt $\left(10^{15} \mathrm{~W}\right)$ interaction facility available to the UK and international user community. The facility came online to users in 2002 and considerable experience has been gained operating the Vulcan facility in this mode. The facility is designed to deliver irradiance on target of $10^{21} \mathrm{~W} \mathrm{~cm}^{-2}$ for a wide-ranging experimental programme in fundamental physics and advanced applications. This includes the interaction of super-high-intensity light with matter, fast ignition fusion research, photon induced nuclear reactions, electron and ion acceleration by light waves and the exploration of the exotic world of plasma physics dominated by relativity.
\end{abstract}

PACS numbers: 42.55.-f, 42.60.-v

(Some figures in this article are in colour only in the electronic version)

\section{Introduction}

In 1998, the science programme based on the Vulcan facility was achieving an unprecedented level of success with high profile scientific publications across a wide range of topics. In particular, the Central Laser Facility (CLF) was recognized as a world-leading centre for high irradiance interaction experiments using its $100 \mathrm{TW}$ [1] chirped pulse amplification (CPA [2]) beamline. In response to user demand, a CLF team devised an upgrade strategy for Vulcan with the aim of delivering Petawatt power from an enhanced CPA beam. The specification is a beam delivery of $670 \mathrm{~J}$ in the stretched pulse delivering $500 \mathrm{~J}$ on target in the compressed pulse of $500 \mathrm{fs}$, giving a peak irradiance of $10^{21} \mathrm{~W} \mathrm{~cm}^{-2}$ in a $5 \mu \mathrm{m}$ focal spot, with a full energy Petawatt shot being fired once every $20 \mathrm{~min}$. This is in comparison to the $2.5 \times 10^{19} \mathrm{~W} \mathrm{~cm}^{-2}$ reported by Kitagawa et al at Osaka University [3] and the $>7 \times 10^{20} \mathrm{~W} \mathrm{~cm}^{-2}$ achieved with a plasma mirror by Perry at Lawrence Livermore National Laboratory (LLNL) [4]. The Petawatt beamline was funded through an Engineering and Physical Sciences Research Council (EPSRC) research grant [5] as the first in a two-step development strategy and was made possible through the transfer of equipment from LLNL following the closure of the Nova laser facility.

The upgrade relies upon important technological developments:

(a) A novel front end amplification architecture based on optical CPA (OPCPA) [6,7] that maintains amplification bandwidth has been designed and commissioned.

(b) An adaptive optics (AO) system has been implemented to improve the beam quality to near diffraction limited. The system is fully functional and low energy measurements in the focal plane show $30 \%$ beam energy delivered within a $5 \mu \mathrm{m}$ spot.

(c) A target chamber has been designed, which combines the requirements for a versatile interaction geometry with the provision of substantial environmental shielding.

\section{Laser commissioning}

The commissioning of the facility was a process of bringing on-line the many individual systems that had been developed off-line and then integrating them into a working facility. The beamline commissioning was complex due to the newly 


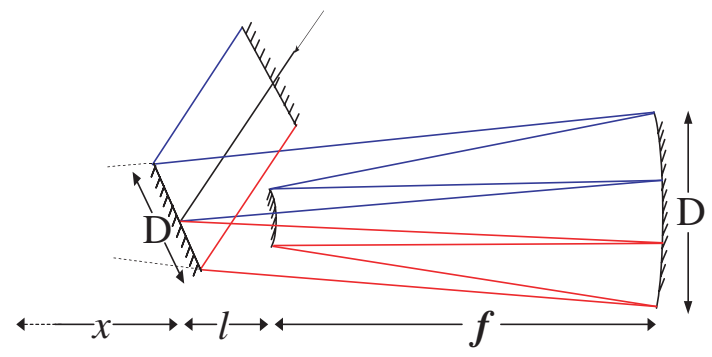

Figure 1. Reflective Offner scheme for the Petawatt upgrade $-f=2250 \mathrm{~mm}, l=625 \mathrm{~mm}, x=3250 \mathrm{~mm}$ and $D=300 \mathrm{~mm}$.

developed systems used, the optical handling required in the compressor and the need to develop new alignment techniques. All of this came together with demonstrations of the required pulse duration, the delivery of laser energy and the required beam quality.

\subsection{Stretcher design}

The CPA technique [2] uses a pulse stretcher prior to amplification followed by a pulse compressor before delivery to target. The degree of stretch is dictated by the need to limit the non-linear self phase modulation (SPM) during the amplification process. The Petawatt design uses a stretch/compression factor in excess of 1000 , and specifies the use of 1480 line $\mathrm{mm}^{-1}$ gratings separated by $13 \mathrm{~m}$.

The stretcher design is based on the use of an Offner triplet optical arrangement. An Offner triplet is a telescope that is formed using two confocal equal positive lenses and one negative lens located at this common focus. The negative lens has half the focal length of either positive lens. There are a number of advantages to using an Offner system. The first is that it forms a 6- $f$ system in comparison to the 4- $f$ Newtonian design. This enables a smaller scale design to be employed and this is of particular use in relation to the Petawatt system. Secondly, if the central negative lens is half the focal length of either positive lens then the system is intrinsically aberration free. This is very important because it allows the use of optics of large aperture (for maximum bandwidth), short focal length (to fit on the 'table top'), and spherical shape (for low cost and ease of manufacture).

The optic layout of the Offner stretcher is shown in figure 1 . The system has been designed to have a maximum bandwidth transmission of $18 \mathrm{~nm}$. This is four and a half times the output bandwidth of Vulcan. A unique feature of this stretcher is the 'double decker' nature of the design. The stretcher is designed using two identical double pass reflective Offner triplets described above, which are located one above the other. This is shown in a side view in figure 2. The incident beam first passes through one system and then the other. The beam is displaced vertically on each pass of the stretcher using retro-reflectors. Using two Offner systems substantially reduces the size and cost of the optics involved and prevents off-axis aberrations that would result from a double pass of a single system.

\subsection{Pre-amplification}

The baseline specification for the Vulcan upgrade is $500 \mathrm{~J}$ delivered to target with pulse duration of $500 \mathrm{fs}$. This

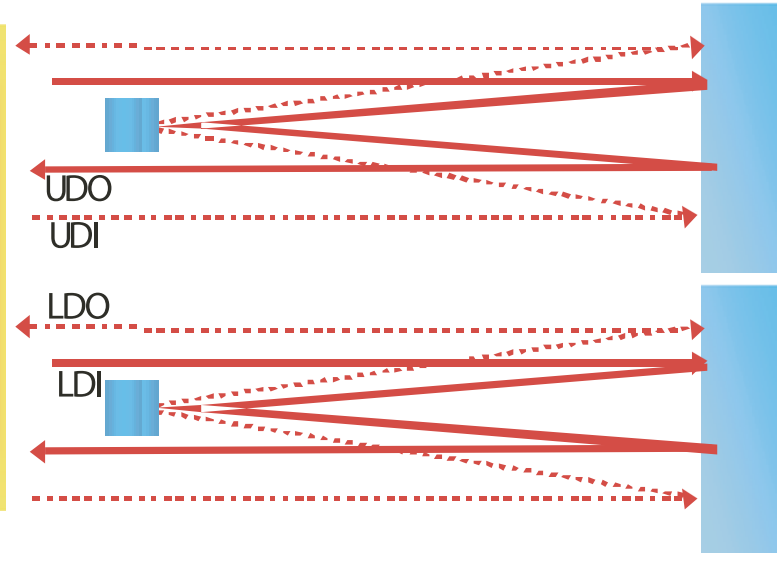

Figure 2. The 'double decker' design — two identical Offner systems sharing a common diffraction grating. Showing the lower decker input (LDI), lower decker output (LDO), the upper decker input (UDI) and the upper decker output (UDO) beams.

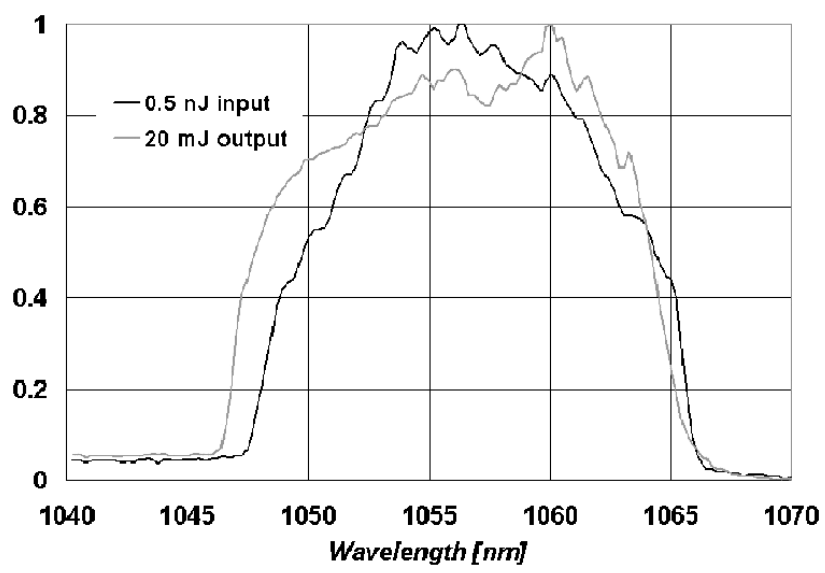

Figure 3. Spectral bandwidth before and after amplification in the OPCPA pre-amplifier demonstrating the potential of the system.

performance is not achievable by amplification solely by $\mathrm{Nd}$ : glass amplifiers, since gain narrowing would reduce the pulse bandwidth to a value below the $\sim 4 \mathrm{~nm}$ limit, which is required to support a $500 \mathrm{fs}$ pulse duration. The approach taken for the Petawatt project was to use an OPCPA pre-amplification system [8], which generates pulses of $\sim 10 \mathrm{~mJ}$ at large bandwidth $\left(1 \mathrm{~mJ} \mathrm{~nm}^{-1}\right)$ for injection into the Vulcan rod and disc amplifier chain. The amplifier chain is optimized for bandwidth using a combination of $\mathrm{Nd}$ :phosphate and $\mathrm{Nd}$ :silicate amplifying media. The pre-amplifier uses a customized $2 \mathrm{~J}, 1053 \mathrm{~nm}, 10 \mathrm{~Hz}$ YAG laser to generate a $4.5 \mathrm{~ns}$ frequency doubled $200-300 \mathrm{~mJ}$ pump pulse which is approximately 'top-hat' in both space and time. This pumps a three-stage optical parametric amplifier that is seeded by the stretched oscillator pulse. The nonlinear medium used for the three amplification stages is $\beta$-barium borate (BBO), each crystal being maintained in an oven at $40^{\circ} \mathrm{C}$.

The bandwidth performance of the OPCPA pre-amplifier is demonstrated in figure 3 . The two curves show the spectrum of the $0.5 \mathrm{~nJ}$ input pulse and the amplified $10 \mathrm{~mJ}$ output. There is no loss of bandwidth with a gain in excess of $10^{7}$. The noise in the input pulse is attributed to the detector used in this measurement and the noise in the amplified output 
pulse is caused by instabilities in the OPCPA pump pulse. High stability of the output energy is an essential criterion for the pre-amplification in order to deliver pulses to target at close to the optical damage limit of the beamline. This requirement is met by operating the third amplification stage in saturation.

During commissioning the OPCPA front-end output demonstrated excellent stability. The occasional drop-out was due to the momentary loss of pump seeding — although no full energy shots have failed due to this mechanism.

\subsection{Amplification}

To provide the $670 \mathrm{~J}$ laser energy required for the Petawatt beamline an additional disc amplifier stage has been commissioned. This comprises three ex-Nova $208 \mathrm{~mm}$ aperture disc amplifiers with gain isolation provided by a $208 \mathrm{~mm}$ aperture Faraday rotator and a single polarizer at the stage input.

One of the $208 \mathrm{~mm}$ amplifiers was modified to operate with four flashlamp circuits using an increased lamp bore to be compatible with Vulcan's existing pulse power system. Its small signal gain was measured to be 2.23 , narrow bandwidth $1053 \mathrm{~nm}$ operation, falling to approximately 2.0 under saturation conditions for $670 \mathrm{~J}$ total output. This performance indicates a saturation fluence of $4 \mathrm{~J} \mathrm{~cm}^{-2}$, in close agreement with the calculated value. This was consistent with the requirements of the Petawatt beamline and all the amplifiers were subsequently modified.

The extended amplifier chain was fully commissioned and the maximum beam energy delivered was $792 \mathrm{~J}$, well in excess of the energy requirements for the beamline. The Faraday rotator has been commissioned and found to give an extinction of $0.5 \%$ operating at $20 \mathrm{kV}$ and triggered $1.8 \mathrm{~ms}$ ahead of the laser pulse.

\subsection{Adaptive optics}

The use of a large aperture, $120 \mathrm{~mm}$, AO module is essential to the Petawatt facility. It is required both to improve the wavefront quality to produce the optimum focal spot on target and also to achieve the best possible recompression of the stretched pulse. The wavefront errors that need to be corrected arise from static aberrations of the optics in the beamline and distortions caused by the thermal gradients set up in the amplifiers during the course of firing laser shots throughout the day. A mirror was designed with 61 addressable elements in a two-dimensional array suitable for correcting these slowly varying aberrations. The deformable mirror is installed after the rod amplifier chain and the wave-front sensor is positioned behind the final disc amplifiers before the Petawatt spatial filter (SF).

The specification for closed loop operation of the AO system for use with Vulcan is for a cycle time $>1 \mathrm{~Hz}$, correction to 1.5 times the diffraction limit and a high level of immunity to electrical noise. Figure 4 shows the far-field intensity distribution of the Vulcan beam with and without the adaptive mirror in place demonstrating the specified beam improvement.

With no phase correction, Vulcan already operates with a beam quality of approximately three times the diffraction limit, and reduction by a factor of 2 gives an increase in irradiance

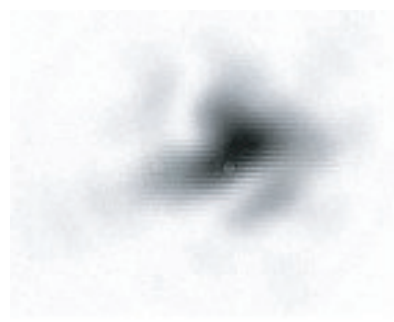

Figure 4. Far-field distribution without (left) and with (right) adaptive correction.

on target of a factor of 4 . This is of significant benefit to the science programme at CLF, the physics of which is very sensitive to irradiance.

\subsection{Compression alignment}

The Vulcan Petawatt specification requires the use of 1480 line $\mathrm{mm}^{-1}$ gratings separated by $13 \mathrm{~m}$. At the compressor end this has been one of the primary drivers dictating the scale of the compression chamber and indeed the actual size of the target area building itself. The layout of the vacuum compressor chamber is shown in figure 5 .

The shortest pulses can only be achieved when phase error has been minimized. The B-integral of the system and any mismatch between the stretcher and the compressor alignment contribute to this. The Vulcan Petawatt system is designed to operate with a B-integral of 1.25 for full energy pulses. The B-integral determines the length of the stretched pulse and cannot be reduced further. An intricate alignment method, therefore, had, to be developed in order to meet the strict tolerances. This involved the use of a two wavelength infrared CW alignment laser positioned before the Petawatt SF, the output of which is shown just before M1 in figure 5 .

The second beamline mirror (M2, see figure 5) was set such that the full aperture $1053 \mathrm{~nm}$ alignment beam propagated back through the compressor system and the Petawatt SF to a far-field monitor. The position of the retro-far-field was recorded as a nominal reference.

The alignment of the first grating was defined geometrically by the positioning of the mounts. The orientation of the grooves was achieved by ensuring that the zeroth- and firstorder reflections were in the same horizontal plane.

The alignment beam was then switched in wavelength to $1047 \mathrm{~nm}$, but had its near- and far-field defined by the same system as for the $1053 \mathrm{~nm}$ beam. The $1047 \mathrm{~nm}$ retrofar-field will be in a different position with respect to the $1053 \mathrm{~nm}$ far-field. This dichroic far-field difference can only be due to a diffractive error. To first order, a horizontal (i.e. in the reference, or dispersion plane) angular dichroic difference is due to a misalignment of grating surface parallelism and requires a relative grating horizontal rotation about a vertical axis to correct. A vertical difference (i.e. perpendicular to the reference plane) is a groove parallelism error and requires a relative grating rotation to correct. This is illustrated in figure 6 . Some care, however, is required in making any correction. The first grating is left unchanged at all times and all adjustments are to the second grating (and M2).

To set the reference (or dispersion) plane grating surface parallelism, small adjustments were made to the second 


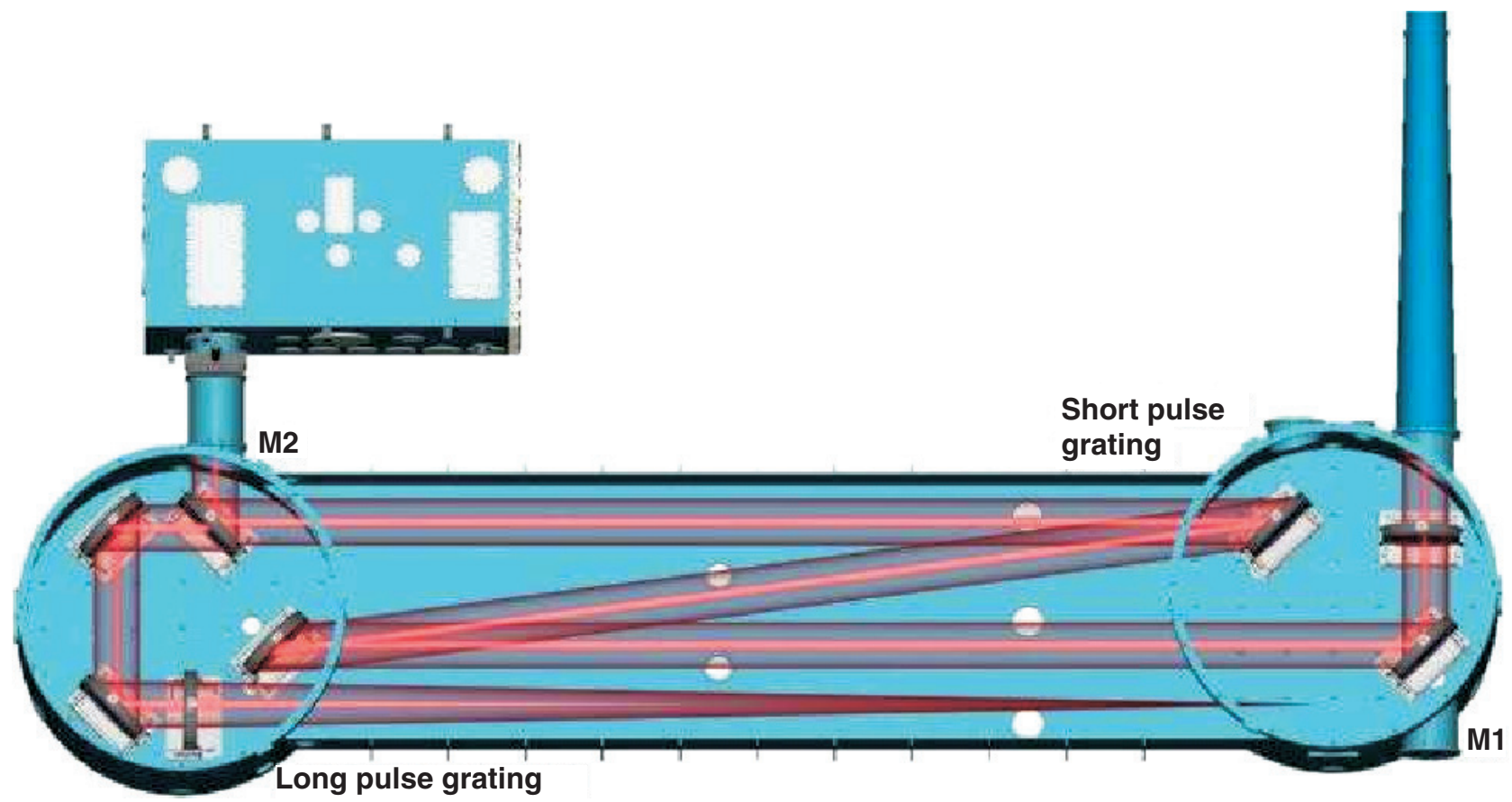

Figure 5. Optical layout of the Petawatt compressor.

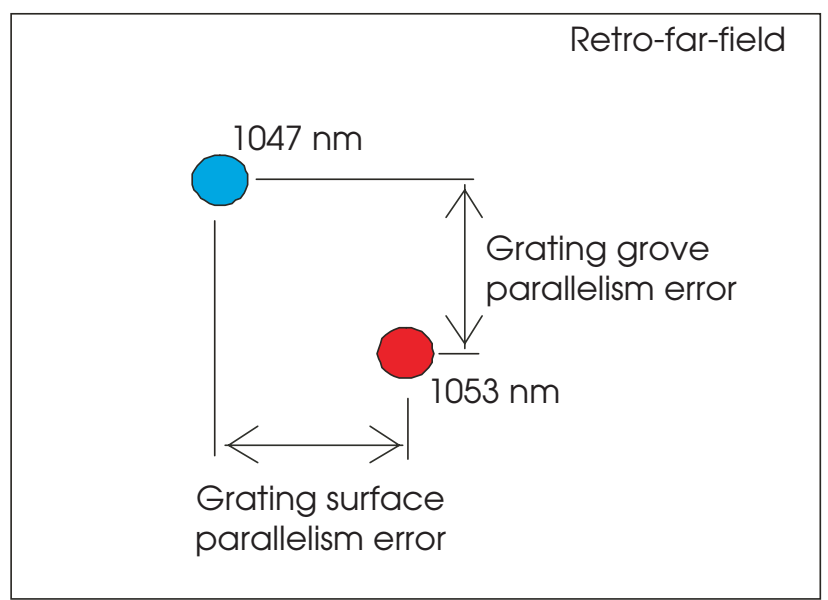

Figure 6. Explanation of retro-far-field dichroic alignment technique.

grating as a horizontal rotation about a vertical axis, whilst always returning the $1053 \mathrm{~nm}$ far-field to the retro-reference position using a corresponding M2 horizontal rotation about a vertical axis adjustment. The dichroic far-field separation was measured and this was repeated until the horizontal dichroic retro-far-field displacement, as determined by a centroiding algorithm, was less than about one-third of a focal spot diameter.

To set the grating groove parallelism small adjustments to the second grating rotation were made, whilst always returning the $1053 \mathrm{~nm}$ far-field to the retro-reference using a corresponding vertical rotation about a horizontal axis adjustment of the second grating (not $\mathrm{M} 2$ as before). This was repeated until the vertical dichroic retro-far-field displacement, as determined by a centroiding algorithm, was also less than one-third of a focal spot diameter. This double pass process will have established that the gratings and their grooves are sufficiently parallel such that in single pass operation there is no residual dispersion across the expected bandwidth that is outside the monochromatic diffraction limit. For this bandwidth, this corresponds to a temporal compression error of no more than $50 \mathrm{fs}$.

To reduce any phase errors due to the mismatch of the compressor and stretcher alignment, the system is optimized for the shortest pulse by using pulses from the OPCPA preamplifier. The OPCPA pulses are used because they are the lowest energy pulses that can be detected with the largest bandwidth at the output of the Petawatt compressor. The exact conjugation of the stretcher/compressor system was performed by injecting the $10 \mathrm{~Hz}$ output of the OPCPA frontend system all the way through the Vulcan chain into the Petawatt compressor. The diagnostics system is capable of operating with this very low (relative) energy OPCPA pulse when M2 is in a near retro, and thus non-HR position.

Initially, as a measure of pulse compression, the diagnostics beam output was focused into a second harmonic crystal. The output of this was maximized by adjusting the longitudinal position of the stretcher grating. Through this method it was possible to determine an approximate position for optimum compression and to bring the pulse length into the window of operation of the two autocorrelators. Finally, there was an optimization of the pulse length by adjusting the stretcher whilst recording the autocorrelator outputs. Given the $10 \mathrm{~Hz}$ operation of the OPCPA system it was possible to do this in real time.

\section{Laser characterization}

Diagnosis of the short pulse on a shot to shot basis is achieved using the $1 \%$ transmission from the $940 \mathrm{~mm}$ diameter mirror 

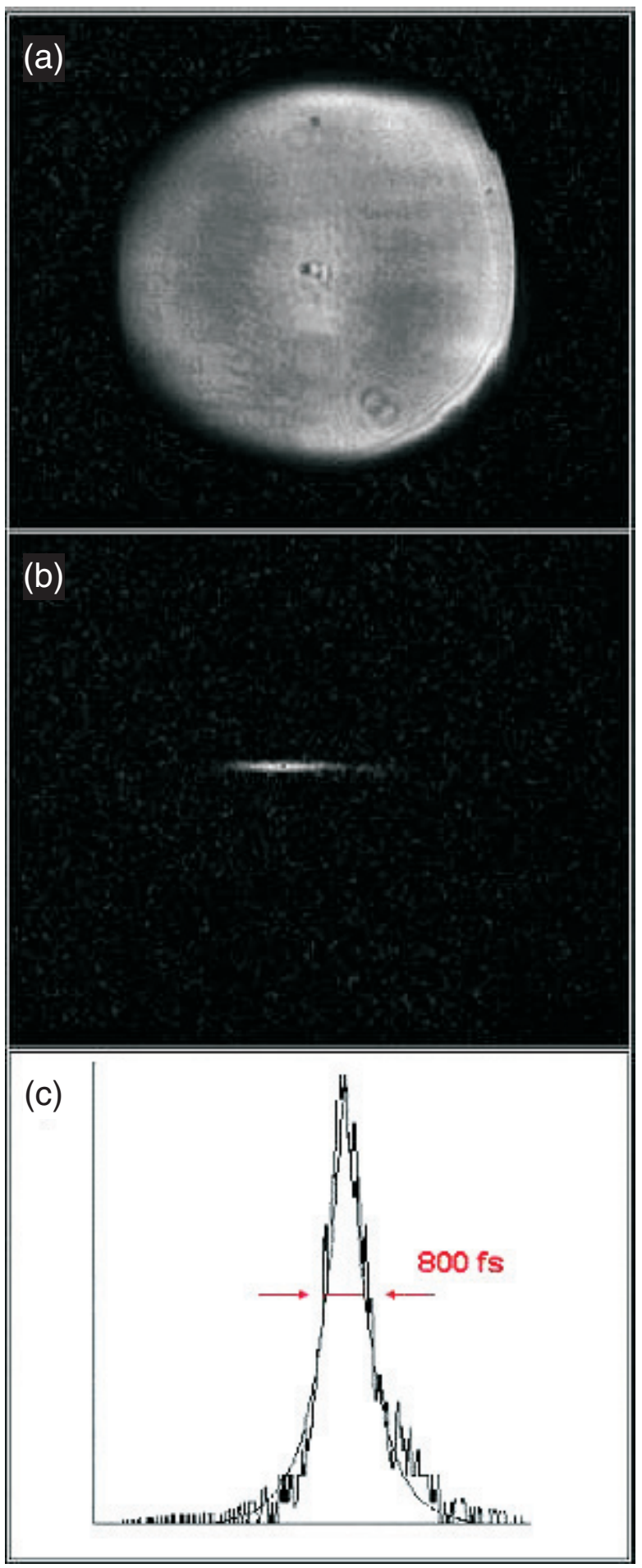

Figure 7. (a) The $1 \%$ transmitted near-field of the compressed pulse; (b) an autocorrelation trace on a $375 \mathrm{TW}$ shot; $(c)$ a line scan through the autocorrelation giving a pulse width of $800 \mathrm{fs}$.

immediately after the second compression grating. Figure 7 gives an example of the compressed pulse characterization of a 375 TW shot. For this shot, the OPCPA pre-amplifier was not fully optimized, giving a reduction in the bandwidth delivered and hence a $\sim 50 \%$ increase in the pulse length delivered. The captured near-field is shown in figure 7(a). The pulse length is monitored using a second-order autocorrelator (figure $7(b)$ )

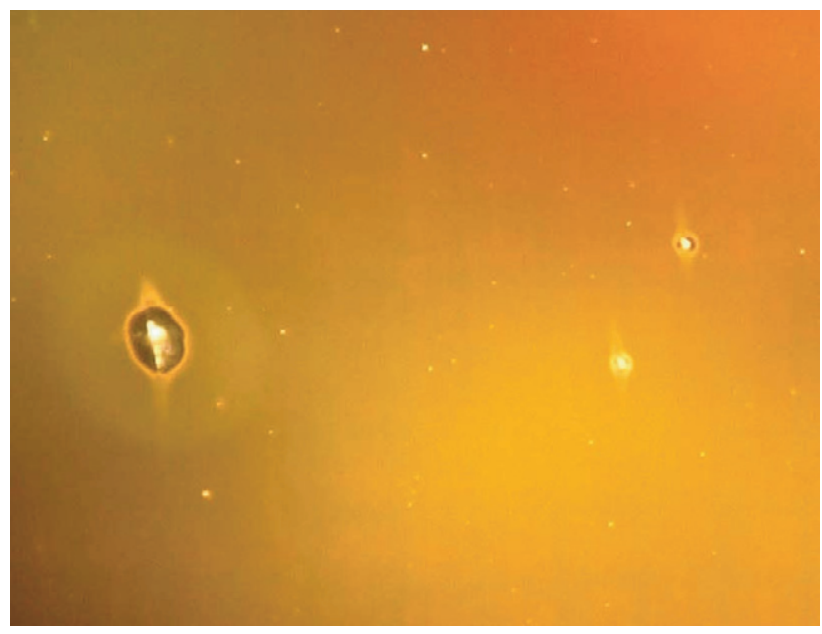

Figure 8. Non-evolutive damage on the second grating.

and the compressed pulse width is measured to be $800 \mathrm{fs}$ (figure 7(c)).

\section{Grating operational experience}

Gold-coated holographic gratings $940 \mathrm{~mm}$ in diameter and with a line density of 1480 lines $\mathrm{mm}^{-1}$ were supplied by LLNL under a 'Work for Others' collaboration. A conservative operational fluence of $170 \mathrm{~mJ} \mathrm{~cm}^{-2}$, to preserve the in-service lifetime of the gratings, and a beam diameter of $600 \mathrm{~mm}$ gave the required operational input energy of $670 \mathrm{~J}$. Beam expansion from the $208 \mathrm{~mm}$ diameter final amplification stage is achieved using a vacuum SF working at F\#25. The expanding SF has an overall length of $19 \mathrm{~m}$.

Following the first shot through the Petawatt system at an energy of $260 \mathrm{~J}$ an inspection was called of all the Petawatt optics. There was concern at the discovey of a large damage mark, that was not recorded before. This mark was found on the second of the two gratings that interacts with the compressed pulse. Upon closer inspection it seemed as if there was a small fissure in either the substrate material or the photoresist layer, which can be seen as a bright vertical line in the centre of the damage site (see figure 8). There appears to be a patch of non-diffractive gold around this site to a diameter of about $3-4 \mathrm{~mm}$, and a larger plume that is less obvious to a diameter of about $12-14 \mathrm{~mm}$.

It is possible to see the fissure in either the substrate or the photoresist from behind although when a torch is shone through no light can be seen in transmission. This seems to indicate that the fissure existed prior to the gold coating of the photoresist.

A number of small sites of damage were detected other than the large spot. Their growth in the horizontal direction has been negligible, although LLNL experience indicates that over a period of time they are likely to grow slowly along the direction of the grating grooves. It is believed that these sites are due to small particles of dust being burnt into the photoresist layer.

Up to the end of 2004 there had been in excess of 200 high power laser shots with regular inspections of the gratings being conducted. The second compressor grating has seen 


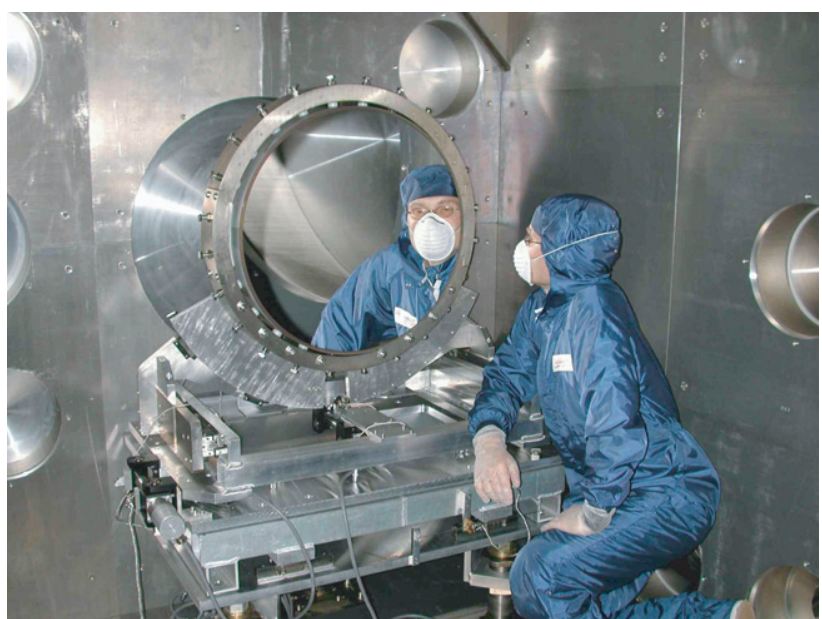

Figure 9. The F\#3.1 off-axis parabola installed in the Petawatt target interaction chamber.

initial damage, which seems to have stabilized, as there is no significant growth in its size or type. The first grating has shown no damage during this period.

\section{Focusing and characterization}

A $620 \mathrm{~mm}$ diameter, $1.8 \mathrm{~m}$ focal length, F\#3.1 off-axis parabola was installed in the Petawatt interaction chamber. The parabola was silver coated with a magnesium fluoride protective overcoat. It is shown during commissioning in figure 9.

Focusing to target and hence optimization of the parabola was conducted using a $1053 \mathrm{~nm} \mathrm{CW}$ alignment laser injected into the beamline at the entrance to the final beam expanding vacuum SF. Injecting the $\mathrm{CW}$ beam at the smaller aperture meant that full aperture alignment could be carried out through the compressor and on to target with modest cost and with confidence that it would be matched to the main laser beam.

The variation of the focal spot diameter was measured as a function of position along the optical axis using a microscope objective and a CCD camera. The result of the $z$-scan for the $\mathrm{CW}$ beam gave a best focal spot size of $5 \mu \mathrm{m}$ FWHM in both the horizontal and vertical scans, which demonstrates the lack of significant astigmatism. Typical examples of the images produced are shown in figure 10 with far-field images in the optimum plane and $80 \mu \mathrm{m}$ either side of this plane.

A scan was also conducted using low energy pulsed beams propagating through the entire amplifier chain which is shown in figure 11. Again, there is little astigmatism and the best focus was found to be $\sim 7 \mu \mathrm{m}$. The slight degradation of the spot observed with the pulsed beam is thought to be due to the broader bandwidth.

It is not possible to maintain adequate filtering when full energy shots are fired and the alternative technique of $\mathrm{x}$-ray imaging was used to measure the emission from the focal region. Figure 12 shows a $20 \mathrm{keV} x$-ray image which has a FWHM of $11 \mu \mathrm{m}$ indicating a smaller optical spot. This was consistent with our most optimistic predictions. Full shot laser far-field measurements were not possible due to optical imperfections in the laser diagnostic chain. An optical compensator has now been designed and will be installed in the system to allow far-field measurements to take place.

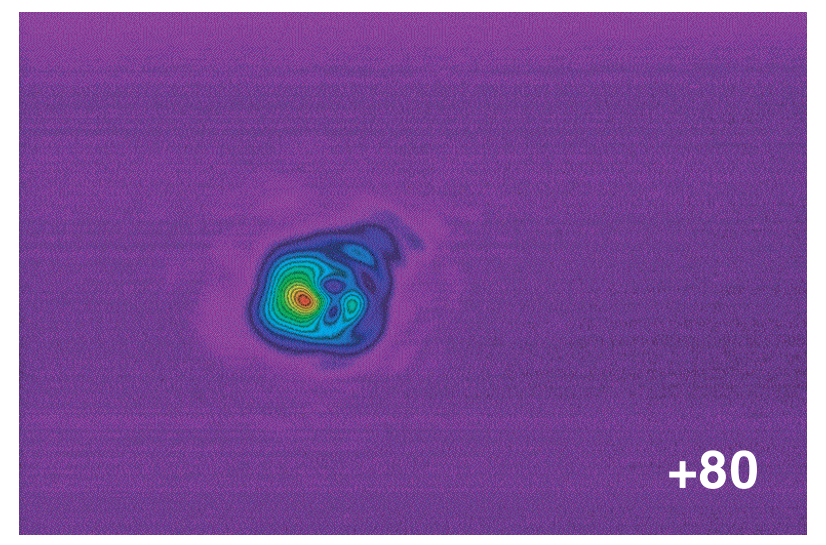

\section{Best focus is 5 mm FWHM}

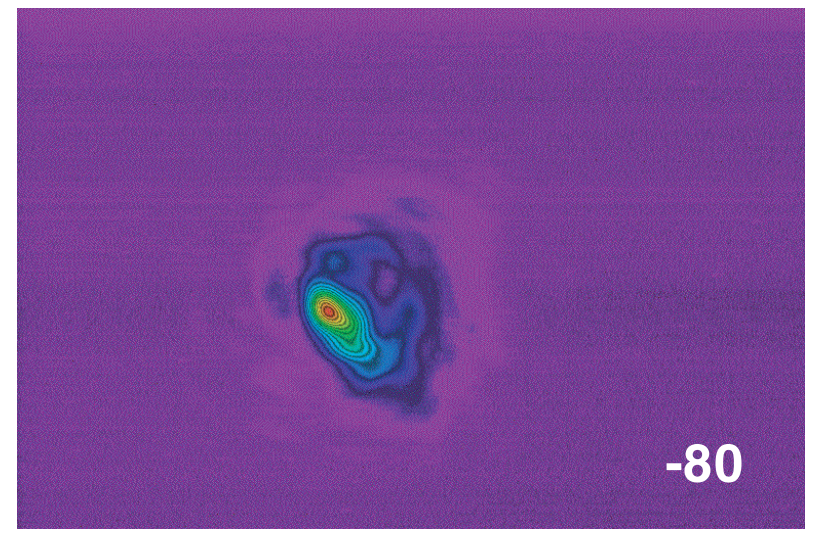

Figure 10. The Vulcan Petawatt focal spot taken with 8-bit CCD using CW alignment laser with $40 \times$ magnification scanned $80 \mu \mathrm{m}$ either side of best focus.

\section{Radiation shielding}

The shielding requirement against radiation ( $\gamma$-rays, $x$-rays, neutrons and activation) produced by Petawatt laser shots on a range of target materials was calculated using experimental electron energy flux distributions and cross checked by scaling data from earlier experiments on Vulcan and other facilities at intensities approaching $10^{20} \mathrm{~W} \mathrm{~cm}^{-2}$. The design required a shield blanket of $15 \mathrm{~cm}$ lead and $10 \mathrm{~cm}$ of high density polyethylene and a shielded fire escape door for medium and high- $Z$ targets.

A photograph of the target area prior to the installation of the shielding around the interaction chamber is shown in figure 13 . 
Vulcan Petawatt

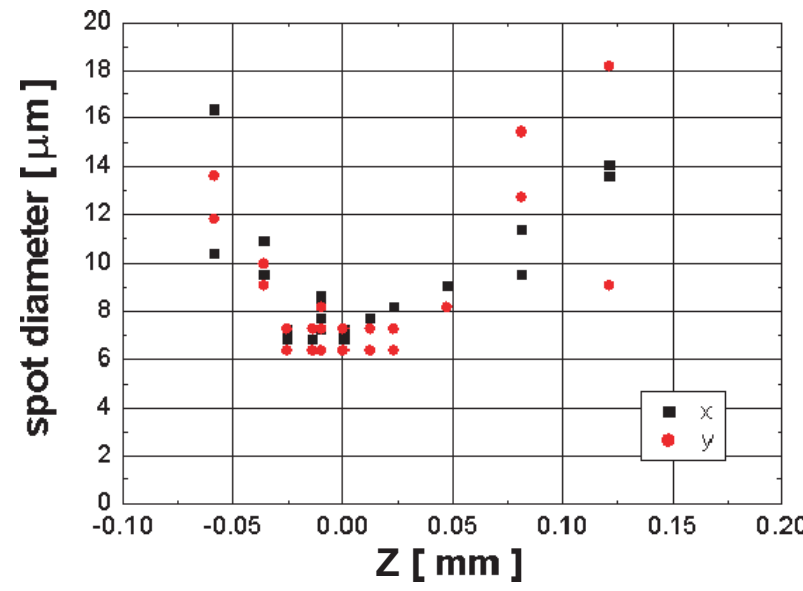

Figure 11. Scan through the focal region of the focusing parabola using low energy pulses from Vulcan.

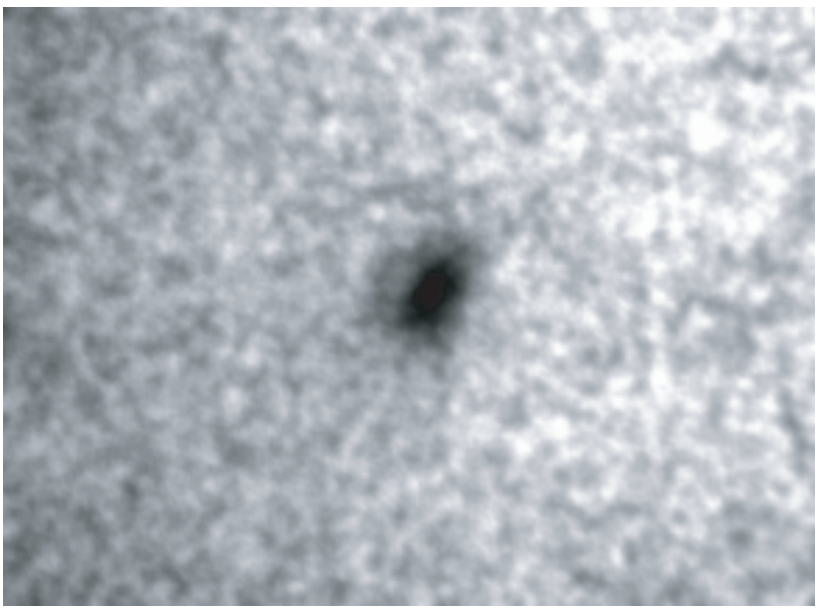

Figure 12. X-ray image of emission from a thin target.

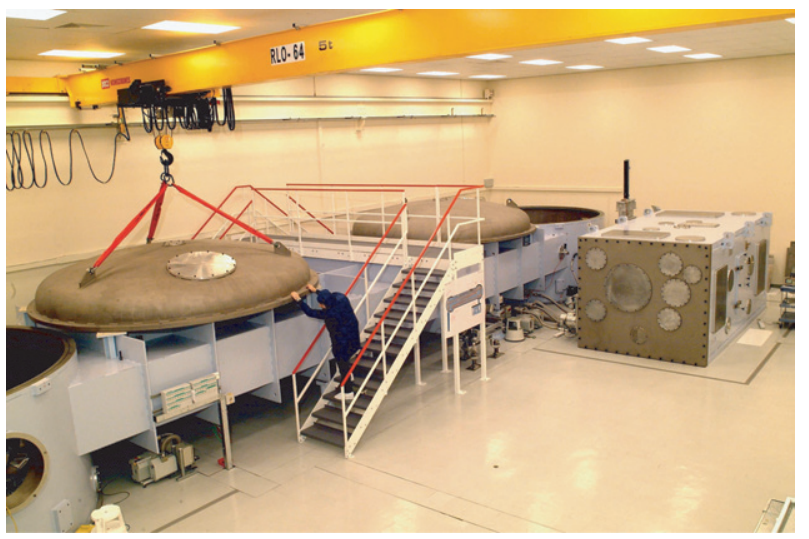

Figure 13. The Petawatt target area prior to the installation of any radiation shielding.

A 70 tonne lead shield wall was installed surrounding the interaction chamber with gaps for access to the diagnostic ports. The $15 \mathrm{~cm}$ thick lead shield wall surrounding the chamber provides high $\gamma$-ray absorption and in combination with the $60 \mathrm{~cm}$ thick concrete walls surrounding the interaction area gives an attenuation of $\gamma$-rays as shown in figure 14 with a minimum direct attenuation of 50000 at

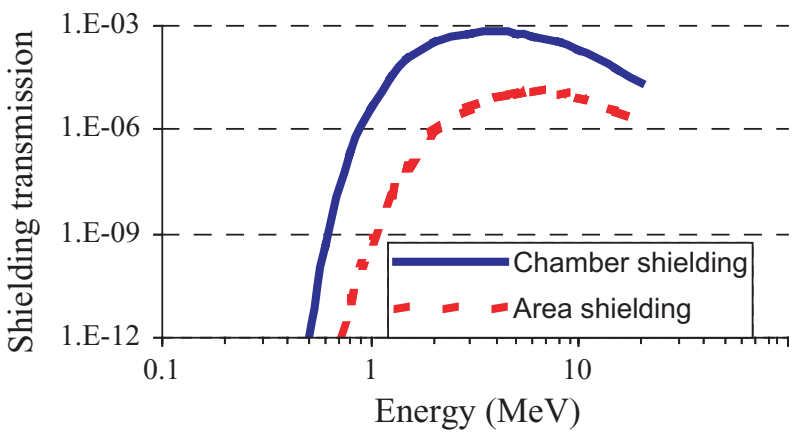

Figure 14. Attenuation of a $\gamma$-ray emission as a function of photon energy.

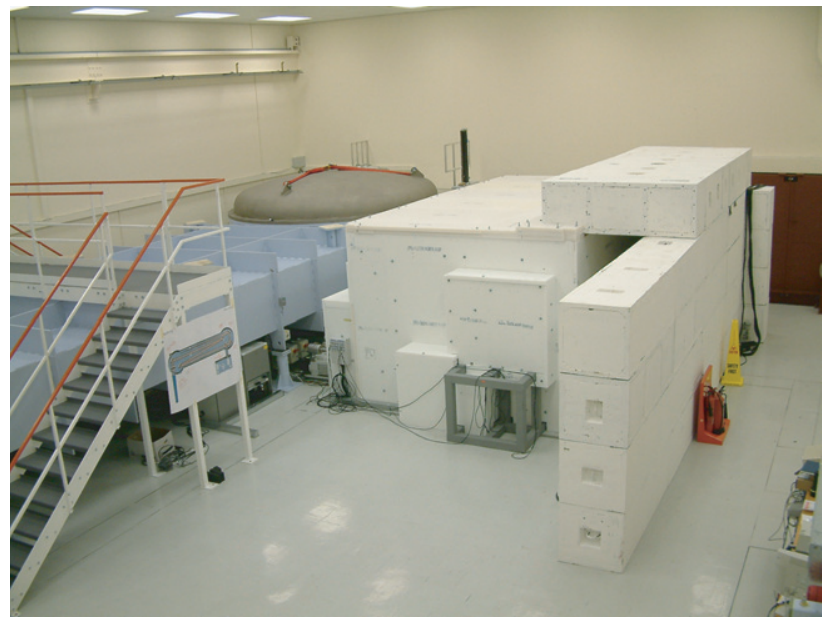

Figure 15. The Petawatt target area complete with radiation shielding.

$6 \mathrm{MeV}$. In addition to the $\gamma$-ray shielding, a neutron absorbing and moderating blanket is required. A $10 \mathrm{~cm}$ thick high density polyethylene shroud was added to the chamber to fulfil this requirement.

Before users could be given access to the newly commissioned facility, a radiological survey had to be conducted to validate the shielding effectiveness and the performance of the focused beam on-target. Radiological dosimetry data demonstrated that the shielding was operating as required. The measurements were approved by the site Radiation Protection Advisor (RPA) for facility delivery to users. During this experiment, significant operational experience was gained in order that the first user experiment could be conducted with a high degree of confidence in the facility's performance. Figure 15 shows the Petawatt target area with the radiation shielding installed around the interaction chamber.

\section{Conclusion}

The Petawatt Interaction Facility at the CCRLC Rutherford Appleton Laboratory has been commissioned and is currently generating up to $500 \mathrm{TW}$ with a focal intensity in excess of $10^{20} \mathrm{~W} \mathrm{~cm}^{-2}$. The ramp-up in power will continue to the Petawatt level and plans for the future development of the facility include the addition of $1 \mathrm{~ns}$ 'long pulse' beams 
and auxiliary probes for target compression, probing and backlighting.

\section{Acknowledgments}

The authors would like to gratefully acknowledge the help of Deanna Pennington, LLNL, USA whose working knowledge of large aperture compressor gratings was invaluable and Prav Patel, LLNL, USA for supplying the data in figure 10.

\section{References}

[1] Danson C.N. et al 1998 Well characterized $10^{19} \mathrm{~W} \mathrm{~cm}^{-2}$ operation of VULCAN-an ultra-high power Nd : glass laser J. Mod. Opt. 451653
[2] Strickland D. and Mourou G. 1985 Compression of amplified chirped optical pulses Opt. Commun. 56219

[3] Kitagawa Y. et al 2004 Prepulse-free petawatt laser for a fast ignitor IEEE J. Quantum Electron. 40281

[4] Perry M.D. 1999 Petawatt laser pulses Opt. Lett. 29160

[5] Hutchinson M.H. 1999 Vulcan Upgrade Project Engineering and Physical Sciences Research Council (EPSRC) Standard Research Grant (GR/M48383)

[6] Dubieties A. et al 1992 Powerful femtosecond pulse generation by chirped and stretched pulse parametric amplification in BBO crystal Opt. Commun. 88437

[7] Ross I.N. et al 1997 The prospects for ultrashort pulse duration and ultrahigh intensity using optical parametric chirped pulse amplifiers Opt. Commun. 144125

[8] Collier J. et al 1999 Evaluation of an ultrabroadband high-gain amplification technique for CPA facilities Appl. Opt. 38 7486 